\section{Denis Tranchand-Bunel Sylvie Jégou Catherine Delbende Marie-Christine Tonon Georges Pelletier Hubert Vaudry}

\title{
La proopiomélanocortine et ses dérivés dans le système nerveux central
}

La proopiomélanocortine est le précurseur de l'ACTH et de divers neuropeptides dont les activités psychotropes sont multiples et parfois antagonistes. Les peptides actifs sont le résultat de diverses réactions de maturation post-traductionnelle qui diffèrent selon les sites de production et de libération.

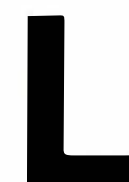

a proopiomélanocortine (POMC) est une glycoprotéine de haut poids moléculaire (31 000 daltons), précurseur multifonctionnel d'une variété de peptides, tels que l'hormone corticotrope (ACTH), les hormones mélanotropes $(\alpha-, \beta$ - et $\gamma$-MSH) et lipotropes ( $\beta$ - et $\gamma$-LPH), et les endorphines $(\alpha-, \beta-$ et $\gamma-$ endorphines) (figure 1). La POMC a d'abord été mise en évidence au niveau de l'hypophyse dans les cellules mélanotropes du lobe intermédiaire et corticotropes du lobe antérieur, puis dans le système nerveux central ( $\mathrm{SNC}$ ). Elle est contenue d'une part, dans les neurones du noyau arqué de l'hypothalamus, et d'autre part, dans la portion commissurale du noyau du tractus solitaire.

Différentes observations ont montré que les peptides dérivés de la POMC, en particulier ceux apparentés à l'ACTH, affectent un certain nombre de comportements [1], ce qui permet d'envi- sager un rôle de neurotransmetteur ou de neuromodulateur pour ces neuropeptides. Cette hypothèse est confirmée par les informations recueillies sur leurs voies de biosynthèse, leur libération en réponse à des stimuli spécifiques, leur inactivation dans la fente synaptique et l'identification de leurs récepteurs dans le SNC.

\section{Structure et biosynthèse de la POMC}

Dès que les structures primaires de l'ACTH et de la $\beta$-LPH ont été déterminées, d'importantes similitudes de séquence entre ces deux molécules ont été remarquées (figure 1). Elles ont en commun un heptapeptide (-Met-GluHis-Phe-Arg-Try-Gly-) qui occupe la position 4-10 dans les molécules d'ACTH et d' $\alpha$-MSH, et 47-53 dans celles de $\gamma$-LPH et $\beta$ LPH. Parallèlement, le développement d'anticorps contre ces peptides a permis de montrer par immunohistochimie en microsco-
G. Pelletier : groupe du MRC en endocrinologie moléculaire, centre hospitalier de l'université Laval, Québec, G1V 4G2, Canada. 


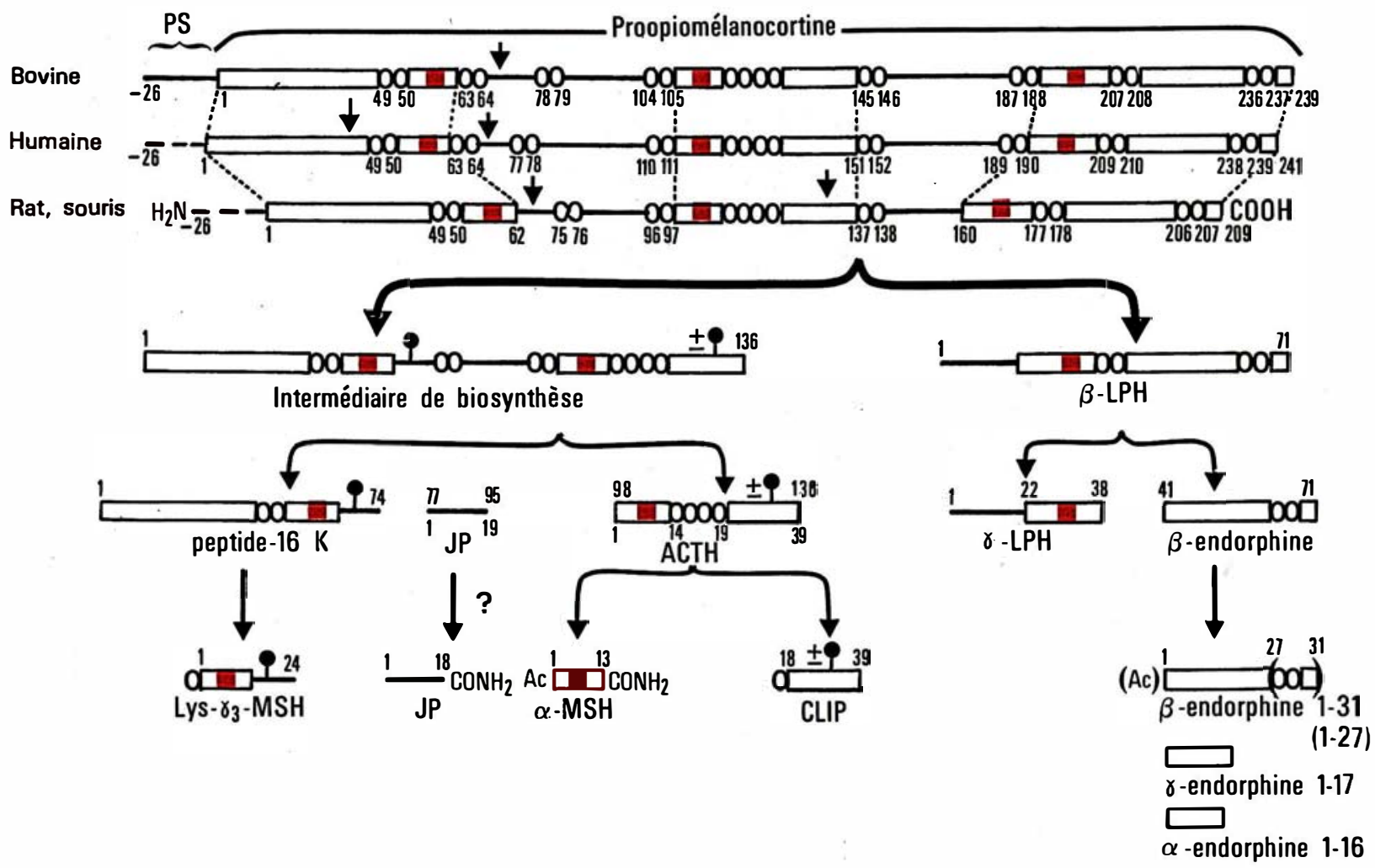

pie électronique que la $\beta$-LPH et l'ACTH sont co-localisées dans les mêmes granules de sécrétion des cellules corticotropes de l'hypophyse du rat [2]. Cet ensemble de données présageait l'existence d'un lien privilégié entre l'ACTH, la $\beta$-LPH et les peptides structurellement apparentés. Les études de biosynthèse réalisées sur une lignée tumorale de cellules antéhypophysaires de souris, AtT-20, ont montré que ces cellules produisent une protéine de masse moléculaire 31000 daltons immunoprécipitable par des anticorps dirigés contre l'ACTH ou la $\beta$-LPH [3]. Cette protéine, $m / s n^{\circ} 3$ ool. 3 , mars 87
Figure 1. Représentation schématique de la structure de la préproopiomélanocortine (pré-POMC) bovine, humaine et de rat, et des voies de biosynthèse des dérivés de la POMC de rat. Les structures des proopiomélanocortines sont déduites des analyses nucléotidiques alors que le schéma de la maturation protélytique est basé sur les études de biosynthèse. L' $\alpha$ $M S H$, les $\beta$ - et $\gamma-M S H$ (bovines et humaines), le CLIP, le JP et les $\beta$-endorphines (1-31, 1-27, 1-26) peuvent potentiellement être libérés par le neurone hypothalamique ou la cellule mélanotrope hypophysaire mais non par la cellule corticotrope hypophysaire. La $\beta$-endorphine peut générer dans la fente synaptique les dérivés 1-17 et 1-16 qui sont respectivement la $\gamma$-et l' $\alpha$-endorphine.

CLIP = corticotropin - like intermediate lobe peptide ;

= portion des trois prohormones ayant des séquences d'acides aminés hautement conservées :

- $=$ portion des trois prohormones ayant des séquences d'acides aminés différentes :

PS = peptide signal de la pré-prohormone;

JP = joining peptide ;

$\downarrow \quad=$ site potentiel de glycosylation;

= chaîne hydrocarbonée ;

O = résidu lysine ou arginine qui constitue des sites de clivage potentiels pour des endopeptidases ;

= heptapeptide (Met-Glu-His-Phe-Arg-Trp-Gly), responsable de l'activité mélanotrope. La structure de cet heptapeptide n'est pas totalement conservée dans la molécule de $\gamma_{3}-\mathrm{MSH}$. 


\section{RÉFÉRENCES}

4. Chrétien M, Benjannet S, Gossard F, et al. From $\beta$-lipotropin to $\beta$-endorphin and " proopiomelanocortin ». Can J Biochem 1979 ; 57 : 1111-22.

5. Roberts JL, Herbert E. Characterization of a common precursor to corticotropin and betalipotropin : identification of beta-lipotropin peptides and their arrangement relative to corticotropin in the precursor synthesized in a cellfree system. Proc Natl Acad Sci USA 1977 ; 74 : 5300-4.

6. Liotta AS, Loudes C, McKelvy JF, Krieger DT. Biosynthesis of precursor corticotropin/endorphin-, corticotropin-, alphamelanotropin-, beta-lipotropin, beta-endorphinlike material by cultured neonatal rat hypothalamic neurons. Proc Natl Acad Sci USA 1980 ; 77 : 1880-4.

7. Nakanishi $S$, Inoue $A$, Kita $T$, et al. Nucleotide sequence of cloned cDNA for bovine corticotropin-beta-lipotropin precursor. Nature 1979 ; 287 : 423-7.

8. Vaudry H, Tonon MC, Delarue C, Vaillant R, Kraicer J. Biological and radioimmunological evidence for melanocyte-stimulating hormone (MSH) of extrapituitary origin in the rat brain. Neuroendocrinology 1978 ; 34 : 9-24.

9. Pelletier G, Leclerc R, Saavedra JM, et al. Distribution of $\beta$-lipotropin $(\beta-\mathrm{LPH})$, adrenocorticotropin (ACTH) and $\alpha$-melanocytestimulating hormone $(\alpha-\mathrm{MSH})$ in the rat brain. I. Origin of the extrahypothalamic fibers. Brain Res 1980 ; 192 : 433-40.

10. Jacobowitz DM, O'Donohue TL. $\alpha$ melanocyte-stimulating hormone : immunohistochemical identification and mapping in neurons of the rat brain. Proc Natl Acad Sci USA $1978 ; 75: 6300-4$.

11. Guy J, Leclerc R, Vaudry H, Pelletier G. Identification of a second category of $\alpha$ melanocyte-stimulating hormone ( $\alpha-\mathrm{MSH})$ neurons in the rat hypothalamus. Brain Res 1980 ; $199: 135-46$.

12. Jégou S, Tonon MC, Guy J, Vaudry H, Pelletier G. Biological and immunological characterization of $\alpha$-melanocyte-stimulating hormone $(\alpha-\mathrm{MSH})$ in two neuronal systems of the rat brain. Brain Res 1983 ; 260 : 91-8.

13. Crine P, Gossard F, Seidah NG, Blanchette L, Lis M, Chrétien M. Concomitant synthesis of beta-END and alpha-MSH from two forms of proopiomelanocortin in the rat pars intermedia. Proc Natl Acad Sci USA 1979 ; 76 : 5085-9.

14. O'Donohue TL, Handelmann GE, Chaconas T, Miller RL, Jacobowitz DM. Evidence that $\mathrm{N}$-acetylation regulates the behavioral activity of $\alpha-\mathrm{MSH}$ in the rat and human central nervous system. Peptides $1981 ; 2$ : 333-44.

15. Zakarian S, Smyth DG. Distribution of active and inactive forms of endorphins in the rat pituitary and brain. Proc Natl Acad Sci USA $1979 ; 76$ : 5972-6.
$31 \mathrm{~K}-\mathrm{ACTH} / \mathrm{LPH}$, précurseur commun de l'ACTH et de la $\beta$ $\mathrm{LPH}$, fût dénommée pro-opiomélanocortine (POMC) [4] en raison de la nature des produits de maturation qui en dérivent et de leurs activités biologiques potentielles de types opiacé, mélanotrope ou corticotrope. Sa structure fût confirmée par l'analyse des produits de traduction des acides ribonucléiques messagers (ARNm) extraits de cellules AtT-20 [5] ou de cellules hypothalamiques de bœuf et de rat [6]. Des études ultérieures sur la séquence nucléotidique de l'ADN complémentaire $(\mathrm{ADNc})$ cloné issu de l'ARNm de la POMC de bœuf [7] ont confirmé et précisé les données structurales obtenues par les méthodes biochimiques conventionnelles [4]. Les produits finaux de maturation de la POMC (ACTH, CLIP, MSHs, LPHs, endorphines) sont délimités par des paires d'acides aminés dibasiques (Lys ou Arg) qui sont des sites de reconnaissance pour les endopeptidases. La pré-POMC possède une séquence "signal " de 26 aminoacides à sa partie $\mathrm{N}$-terminale. Son caractère hydrophobe conditionne sa translocation dans le lumen du réticulum endoplasmique rugueux, étape nécessaire à la sécrétion d'un polypeptide, entraînant à sa suite la protéine naissante $\left(\mathrm{m} / \mathrm{s} n^{\circ} 6\right.$, vol 2, p. 341). Ce peptide signal est rapidement clivé par une signal-peptidase, véritable première enzyme de maturation, détachant la séquence peptide signal de la POMC.

\section{Distribution dans le système nerveux central}

Les premières études révélant la présence d' $\alpha$-MSH dans le diencéphale ont été réalisées soit par quantification de l'activité mélanotrope soit par dosage radioimmunologique du peptide [8]. Ces résultats sont en accord avec les données immunohistochimiques qui montrent que les péricaryons contenant l' $\alpha$-MSH, l'ACTH et les autres dérivés de la POMC sont principalement localisés dans l'hypothalamus [9]. Par la suite, les études immunohistochimiques ont révélé l'existence de trois systèmes neuronaux producteurs de peptides dérivés de la POMC chez le rat (figure 2). Le premier, situé dans le noyau arqué de l'hypothalamus, produit l' $\alpha$-MSH et ses dérivés, la $\gamma$-MSH et la $\beta$ endorphine. Ces neurones projettent vers les aires pré-optique et hypothalamique antérieure, vers le septum et le thalamus, latéralement vers l'amygdale et postérieurement vers les régions du cerveau moyen et postérieur [10]. Le second système neuronal localisé dans la région dorso-latérale de l'hypothalamus (zona incerta) [11, 12] ne produit que l' $\alpha$-MSH monoacétylée [12]. Les corps cellulaires de cette région projettent des fibres dans le cortex cérébral, l'hippocampe et le striatum. Enfin, un troisième groupe de péricaryons à $\mathrm{POMC}$, récemment mis en évidence dans le noyau du tractus solitaire, projette ses fibres localement dans le mésencéphale et vers la moelle épinière.

Les études de biosynthèse de l'ACTH par des neurones hypothalamiques en culture [6] et la mise en évidence de l'ARNm de la POMC dans le cerveau par Northern Blot (voir $m / s n^{\circ} 9$, vol 2, p. 518) et hybridation in situ, montrent que les peptides du groupe POMC localisés par immunohistochimie et quantifiés par radioimmunologie [8] au niveau du cerveau ont une origine intrinsèque.

\section{Maturation de la POMC cérébrale}

La maturation de la POMC s'effectue dans les vésicules synaptiques au départ des péricaryons et lors du transport axonal. Des endopeptidases ou prohormoneconverting enzymes (PCEs), qui ne sont vraisemblablement pas de type trypsique, assurent en milieu acide $(\mathrm{pH} 5,4)$ le clivage de la POMC au niveau des paires d'acides aminés basiques (figure 4). La présence ou non de ces PCEs, chacune étant, semble-t-il, spécifique de l'enchaînement des doublets basiques, conditionnerait la protéolyse plus ou moins poussée 
de la POMC. Cette hypothèse permet d'expliquer que dans les cellules corticotropes de l'adénohypophyse, la POMC est clivée en $\beta$-LPH, ACTH, peptide $16 \mathrm{~K}$ et, accessoirement, en $\beta$-endorphine, alors que dans les cellules mélanotropes du lobe intermédiaire et dans l'hypothalamus l'équipement enzymatique des cellules permet à la maturation de se poursuivre [13]. L'ACTH est alors fragmentée en $\alpha$-MSH et CLIP (corticotropin-like intermediate lobe peptide), tandis que la $\beta$ LPH sert de précurseur à la $\gamma$ $\mathrm{LPH}$ et à la $\beta$-endorphine qui peut, à son tour, générer un certain nombre de dérivés : les $\boldsymbol{\gamma}$ - et $\alpha$-endorphines et leurs formes destyrosylées. Des exopeptidases de type $B$, moins spécifiques, enlèvent respectivement les résidus $\mathrm{N}$ et/ou C-terminaux laissés par la ou les PCEs. Toutefois, d'autres enzymes participent à la formation de peptides qui, telles les $\alpha$ - et $\gamma$ endorphines, ne sont pas délimités par des paires d'acides aminés basiques. Ces activités enzymatiques de type carboxypeptidasique qui ont été identifiées dans le cerveau seraient liées à la membrane synaptique et agiraient après libération de la $\beta$-endorphine. Enfin, d'autres activités enzymatiques non peptidasiques sont également impliquées dans la maturation des dérivés de la POMC. Les quatre composantes essentielles de cette maturation post-traductionnelle sont : la glycosylation, la phosphorylation, l'acétylation et l'amidation.

Chez toutes les espèces étudiées, la POMC possède un site préférentiel de glycosylation sur le résidu 65 (Asn) du peptide $16 \mathrm{~K}$ correspondant à la séquence : Asn-X-Ser. Le fragment $16 \mathrm{~K}$ humain présente en outre une $\mathrm{O}$ glycosylation de la thréonine en position 45. Chez le rat et la souris, une partie de la POMC est également glycosylée en position 29 de l'ACTH. Ces glycosylations ont lieu dans le réticulum endoplasmique et protègent la molécule d'une protéolyse non spécifique in situ. Le rôle physiologique de la phosphorylation de l'ACTH sur la $m / s n^{\circ} 3$ ool. 3, mars 87 sérine en position 31 , s'il existe, reste encore obscur. L'acétylation est également une étape importante dans la maturation de l' $\alpha$ MSH et des endorphines. Dans le cerveau, l' $\alpha$-MSH peut être présente sous trois formes : déacétylée, monoacétylée $(\alpha-\mathrm{MSH}$ authentique) et diacétylée en positions $\mathrm{N}$ - et O-terminales. De même, les $\beta$-endorphines $1-31$ et 1-27 existent sous les formes $\mathrm{N}$ acétylée et surtout déacétylée. $\mathrm{La}$ même enzyme appelée opiomelanotropin acetyltransferase (OMAT) acétyle à la fois l' $\alpha-\mathrm{MSH}$ et la $\beta$ endorphine en utilisant l'acétylcoenzyme A comme substrat. Il est intéressant de noter que l'acétylation augmente l'activité mélanotrope et les effets comportementaux de l' $\alpha$-MSH [14] alors que sous la forme acétylée, la $\beta$ endorphine et ses dérivés perdent leur activité opiacée [15]. L'acétylation semble donc être un moyen de contrôle de l'activité biologique. De fait, des études récentes ont montré que certains facteurs, telle la dopamine, impliqués dans le contrôle de la libération des dérivés de la POMC extracérébrale, sont également susceptibles d'agir sur le niveau d'acétylation de l' $\alpha$-MSH [16] et de la $\beta$ endorphine [17] ou sur l'activité de l'acétyltransférase. L'amidation C-terminale de l' $\alpha$-MSH est aussi très importante pour son activité mélanotrope. De nombreux peptides régulateurs possèdent un groupement carboxyl-C-terminal $\alpha$-amidé : l' $\alpha$-MSH, l'ocytocine, la vasopressine, la substance $P$, la cholécystokinine, la gastrine, la sécrétine, le peptide intestinal vasoactif (VIP), les peptides de la famille du neuropeptide Y (NPY) et les releasing-factors hypothalamiques (LH-RH, GH-RH, TRH...) [18]. L'analyse des différents précurseurs des peptides $\alpha$ amidés montre qu'ils contiennent tous la séquence -X-Gly-basicbasic-. La paire d'amino-acides basiques (Lys ou Arg) marque le site de clivage protéolytique de maturation. Le fragment Cterminal du peptide alors généré, -X-Gly-COOH, sert de substrat à l'enzyme d'amidation qui produit le peptide $\alpha$-amidé, $-\mathrm{X}-\mathrm{CONH}_{2}$, où la fonction amide provient de la glycine. L'enzyme d'amidation a été caractérisée dans les grains de sécrétion de l'hypophyse de bœuf, de porc, de rat et de souris. Récemment, une enzyme qui présente des caractéristiques similaires a été isolée dans les vésicules synaptiques de l'hypothalamus de rat (figure 4). L'activité de cette enzyme dépend de la présence des ions cuivre et d'acide ascorbique. La réaction nécessite également de l'oxygène moléculaire. Il a donc été proposé pour cette cuproenzyme le nom de peptidylglycine $\alpha$ amidating monooxygenase ou PAM. L'amidation de l' $\alpha$-MSH se fait selon le schéma classique décrit plus haut. On remarque qu'en utilisant un modèle de culture primaire de cellules mélanotropes qui présentent la propriété de perdre leur activité d'amidation au cours du temps, le résidu Gly ${ }^{14}$ n'est pas supprimé dans le précurseur ACTH (1-14)OH (Ac-Ser ${ }^{1}$-.. $\left.\mathrm{Val}^{13}-\mathrm{Gly}^{14}-\mathrm{OH}\right)$ de l' $\alpha$-MSH, et que seule l'amidation permet de générer l' $\alpha$-MSH biologiquement active. L'analyse nucléotidique du gène de la POMC permet de prévoir trois sites d'amidati on qui, s'ils sont reconnus par li PAM, peuvent conduire à la formation de l' $\alpha$-MSH, $\gamma_{1}-\mathrm{MSH}$ e $j$ ining peptide (JP) (figure 1). Com te tenu du rôle important que juz en général l'amidation dans I'activité biologique de nombreux neuropeptides, on peut envisager que ces deux derniers peptides puissent exercer des effets psychotropes encore inconnus.

\section{Régulation de l'activité des neurones à POMC}

a) Bases anatomiques. La structure complexe du noyau arqué où prennent naissance les fibres à POMC mérite une attention particulière. Du point de vue anatomique, le noyau arqué entoure la partie ventrale du troisième ventricule et est composé d'une population dense de petits neurones arborant plusieurs dendrites qui lui conferent un rôle de centre 


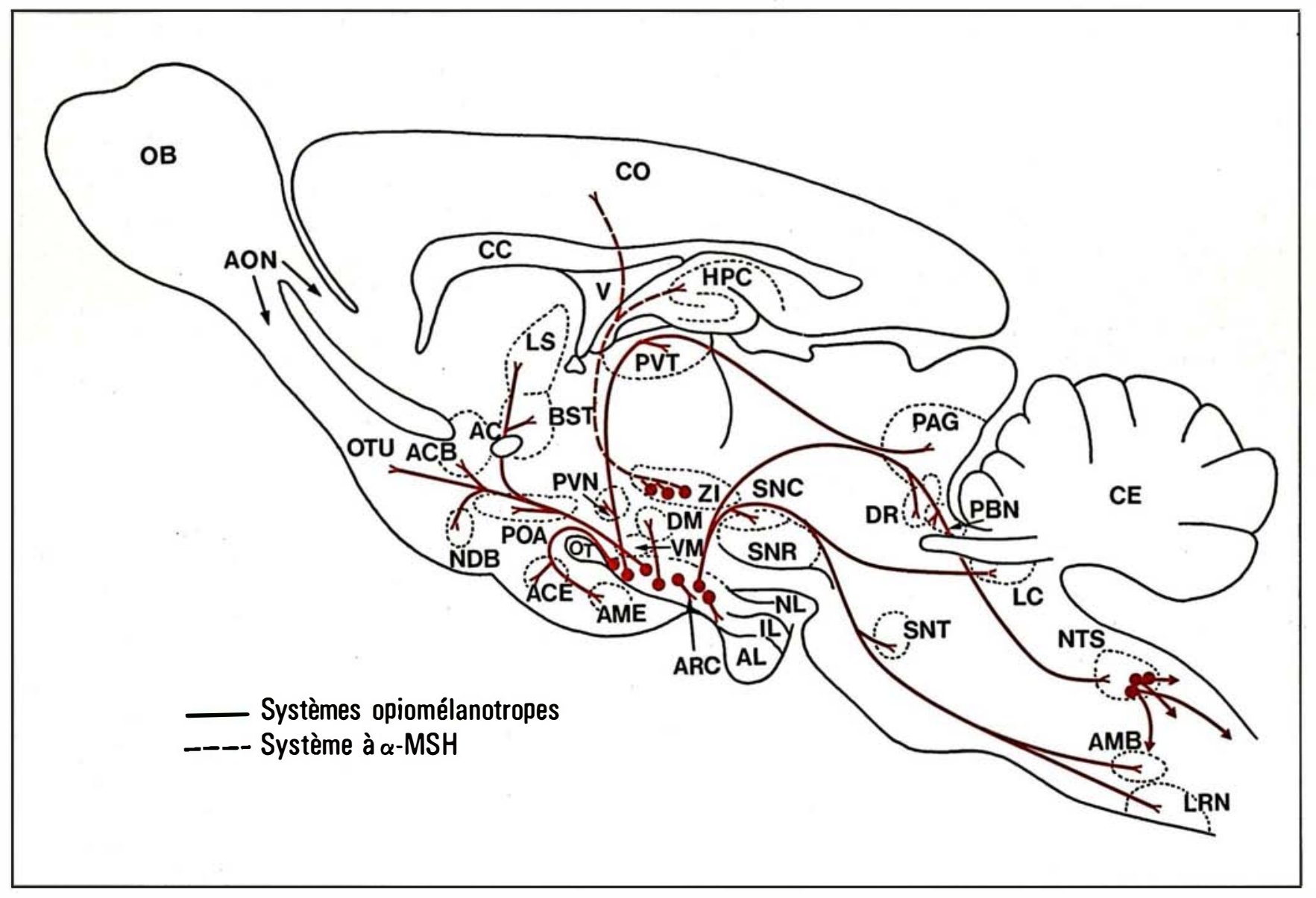

Figure 2. Systèmes opiomélanotropinergiques et mélanotropinergique dans lo cerveau do rat. Les cellules opiomélanotropes du noyau arqué (ARC) et du noyau du tractus solitaire (NTS) synthétisent à la fois les peptides opiacés et mélanocorticotropes. Dans le cas du noyau arqué, les neurones à POMC projettent dans différentes aires du cerveau (lignes rouges continues), en particulier antérieurement vers le thalamus (PVT), le septum latéral (LS), le noyau accumbens (ACB) et le noyau du lit de la stria terminalis (BST), latéralement vers l'amygdale (AME, AMB) et postérieurement vers le cerveau moyen (PAG, $D R$ ) et postérieur (LC, NTS). Les neurones mélanotropes de la zona incerta (ZI) synthétisent uniquement I' $\alpha$-MSH et projettent dans le cortex (CO) et I'hippocampe dorsal (HPC) (lignes rouges discontinues).

\section{NOYAUX}

$A C=$ anterior commissure

ACB = nucleus accumbens

ACE = central nucleus of amygdala

AHA = anterior hypothalamic area

$A L=$ anterior lobe of the pituitary

AMB = nucleus ambiguus

AME = medial nucleus of amygdala

AON = anterior olfactory nucleus

ARC = arcuate nucleus

BST = bed nucleus of the stria terminalis

CC $=$ corpus callosum

CE $=$ cerebellum

CO $=$ cortex

DM = dorsomedial nucleus of hypothalamus

DPM = dorsal premammillary nucleus

DR = dorsal raphe nucleus

HPC = hippocampus

IL = intermediate lobe of the pituitary

LC = locus coeruleus

LRN = lateral reticular nucleus

$\mathbf{L S}=$ lateral septum

NDB = nucleus of diagonal band

$N L=$ neural lobe of the pituitary
NTS = nucleus of the tractus solitarius

OB = olfactory bulb

OT $=$ optic tract

OTU = olfactory tubercle

PAG = periaqueductal gray

PBN = parabrachial nucleus

POA = preoptic area

PVN = paraventricular nucleus

PVT = periventricular nucleus of the thalamus

$\boldsymbol{R C}=$ retrochiasmatic nucleus

$\boldsymbol{R F}=$ reticular formation

SC = suprachiasmatic nucleus

SNC = substantia nigra (pars

compacta)

SNR = substantia nigra (pars reticulata)

SNT = sensory nucleus of trigeminal $\mathbf{S T}=$ stria terminalis

$\mathbf{V}=$ ventricle

$V M=$ ventromedial nucleus of hypothalamus

$\mathbf{Z I}=$ zona incerta 


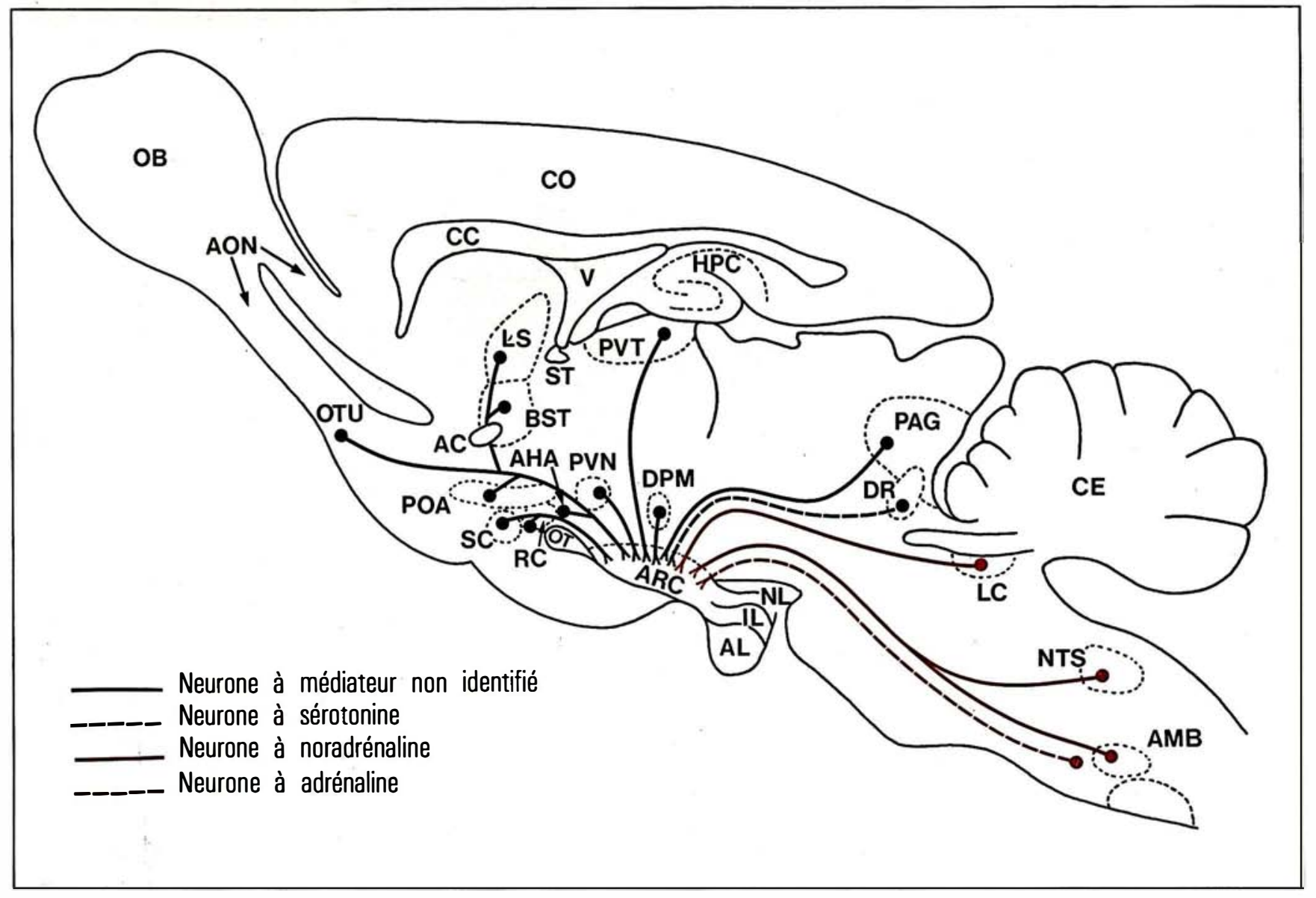

Figure 3. Afférences de I'hypothalamus médio-basal (ARC). De manière générale, il semble que le noyau arqué reçoive des afférences des noyaux qu'il contrôle (voir figure 2). L'organisation privilégiée du noyau arqué le désigne comme un centre intégrateur modulant les réponses d'un nombre considérable de noyaux du cerveau impliqués dans l'émotion (système limbique), le sensoriel et la douleur (thalamus et substance grise mésencéphalique), les fonctions végétatives (hypothalamus) et autonomes (bulbe). intégrateur d'informations. D'une part, il envoie des projections vers l'éminence médiane sous-jacente et communique avec l'hypophyse par voies neuronale et humorale. D'autre part, il reçoit du SNC des afférences intrahypothalamiques des aires médio-basale, antérieure et latérale, du noyau ventromédian, de l'aire "préoptique, mais aussi extrahypothalamiques issues des diencéphale, mésencéphale et télencéphale (figure 3). Divers neurotransmetteurs classiques et neuropeptides pourraient donc jouer un rôle dans la régulation de la synthèse de la POMC et la libération de ses dérivés. Des études immunocytochimiques indiquent que des fibres sérotoninergiques en provenance du noyau du raphé se projettent dans l'hypothalamus en général et en particulier au niveau des péricaryons à POMC $[19,20]$. Le locus coeruleus, le tractus solitaire et la formation réticulée produisent, de leur côté, un réseau de fibres noradrénergiques [21] qui projettent vers le noyau arqué. De manière générale, il semble que celui-ci reçoive des afférences des noyaux qu'il contrôle (figures 2 et 3). Deux systèmes aminergiques intrahypothalamiques inhibiteurs de la cellule mélanotrope hypophysaire chez les mammiferes sont également localisés dans le noyau arqué de la souris et du rat : il s'agit des corps cellulaires à dopamine et à acide $\gamma$ aminobutyrique. Des études morphologiques montrant des collatérales récurrentes [22] sur les neurones à POMC suggèrent l'existence possible d'une autorégulation. Enfin, trois systèmes peptidergiques bordant les cellules à POMC ont été identifiés dans le noyau arqué : substance $P$, enké- 


\section{RÉFÉRENCES}

16. Jenks BG, Van-Kemenade BML, Tonon MC, Vaudry H. Regulation of biosynthesis and release of pars intermedia peptides in Rana ridibunda : dopamine affects both acetylation and release of $\alpha-\mathrm{MSH}$. Peptides 1985 , 6 : 913-21.

17. Ham J, Smyth DG. $\beta$-endorphin processing in pituitary and brain is sensitive to haloperidol stimulation. Neuropeptides 1985 ; 5 : 497-500.

18. Mains RE, Eipper BA, Glembotski CC, Dores RM. Strategies for the biosynthesis of bioactive peptides. Trends Neurosci 1983 ; 6 : 229-35.

19. Kiss J, Leranth CS, Halasz B. Serotoninergic endings on VIP-neurons in the suprachiasmatic nucleus and on ACTH-neurons in the arcuate nucleus of the rat hypothalamus. A combination of high resolution autoradiography and electron microscopic immunocytochemistry. Neurosci Lett 1984 ; 44 : 119-29.

20. Bosler O, Beaudet A. Relations structurales entre systèmes monoaminergiques et peptidergiques dans l'hypothalamus. Ann Endocrinol (Paris) 1985 ; 46 : 19-26.

21. Palkovits M, Zaborszky L, Feminger A, et al. Noradrenergic innervation of the rat hypothalamus : experimental biochemical and electron microscopic studies. Brain Res 1980 ; 191 : 161-71.

22. Chen YY, Pelletier G. Demonstration of contacts between proopiomelanocortin neurons in the rat hypothalamus. Neurosci Lett 1983 ; 43 : 271-6.

23. Pique L, Jégou $S$, Bertagna $X$, et al. Proopiomelanocortin peptides in the human hypothalamus. Comparative study between normal subjects and parkinson patients. Neurosci Lett 1985 ; $54: 141-6$.

24. Van Ree JM, Gaffori O. Uniquely, $\boldsymbol{\gamma}$ endorphin induces an effect that is both opiatelike and neuroleptic-like. Life Sci 1983 ; 33, Supp 1 : 619-22.

25. De Wied D, Jolles J. Neuropeptides derived from proopiomelanocortin on behavioral physiological and neurochemical effects. Physiol Reo 1982 ; 62 : 976-1059.

26. Labrie F, Dupont A, Cusan L, Ferland L, Coy DH, Li CH. Effects of endorphins and their analogues in neuroendocrine control. In : Usdin E, Bunny W, eds. Endorphins in Menlal Health Research. New York : Oxford University Press, 1979.

27. Van Praag HH, Verhoeven VMA, van Ree JM, De Wied D. The treatment of schizophrenic psychoses with gamma-endorphins. Biol Psychiatry $1982 ; 17: 83-98$.

28. Van Ree JM, Bohus B, Csontos KM, et al. Behavioral profile of gamma MSH relationship with ACTH and $\beta$-endorphin action.

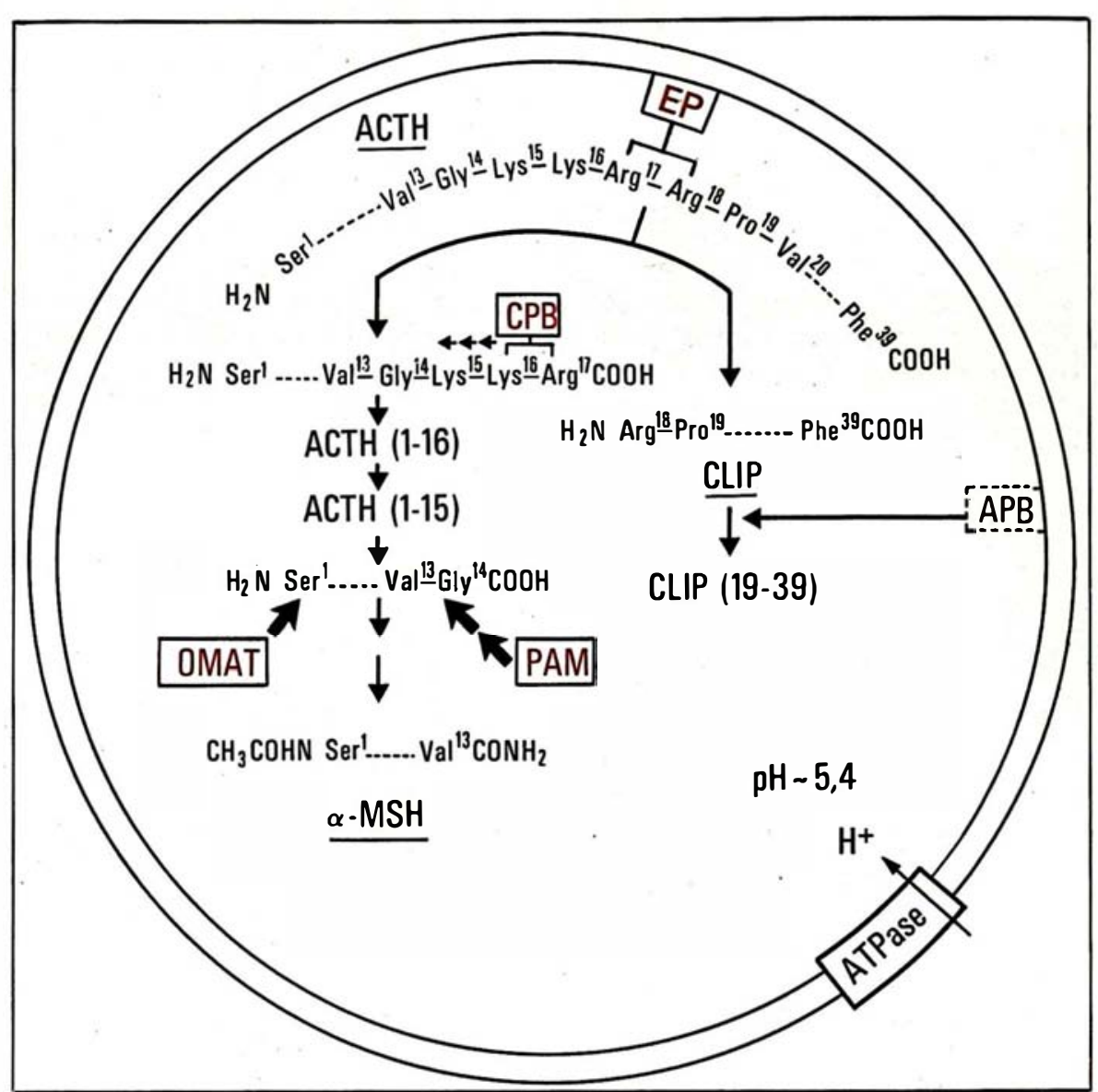

Figure 4. Représentation schématique des principales étapes onzymatiques de maturation posttraductionnelle de la proopiomélanocortine (POMC) survenant dans une vésicule synaptique. La maturation pourrait commencer dans l'appareil de Golgi avec le clivage protéolytique au niveau de certaines paires de résidus basiques par une "prohormone converting enzyme " (PCE) qui est une acid-thiol-arginyl protéase. Elle se poursuit au cours du transport axonal, dans les vésicules synaptiques par l'action d'endopeptidases (EP) de la famille des PCE, a partir des fragments de la POMC alors générés (ACTH, $\beta-L P H$, etc.). Les carboxypeptidase (CPB) et/ou aminopeptidase (APB) de type $B$, moins spécifiques, clivent ensuite respectivement, les résidus basiques $C$ - et/ou $\mathrm{N}$-terminaux laissés par l'endopeptidase (EP). Certains peptides clivés, comme l' $\alpha-M S H$, subissent des transformations, telles que $I^{\prime} \alpha$ - $N$-acétylation assurée par I'" opiomelanotropin-acetyltransferase " (OMAT) et l' $\alpha$-amidation $C$ terminale par la "peptidyl-glycine $\alpha$ amidating monooxygenase " (PAM). phalines et neuropeptide Y. Compte tenu de la complexité de l'organisation du noyau arqué et de la multiplicité des médiateurs mis en jeu, on peut s'attendre à ce que les neurones hypothalamiques à POMC soient soumis à un contrôle plurifactoriel.

b) Régulations transcriptionnelle et traductionnelle. Chez le rat, la transcription du gène de la POMC dans l'hypothalamus, n'est pas altérée par les glucocorticoïdes, la surrénalectomie ou l'administration d'agonistes dopaminergiques. Au niveau hypophysaire, les glucocorticoïdes circulants inhibent la transcription du gène de la POMC dans les cellules corticotropes antéhypophysaires mais ne modifient pas la vitesse de transcription de la POMC dans les cellules mélanotropes du lobe intermédiaire. En revanche, la dopamine inhibe la transcription du gène de la POMC au niveau du lobe intermédiaire. Il 
apparaît donc que les systèmes de régulation de l'expression génomique de la POMC sont tout à fait différents dans les neurones et les cellules épithéliales.

En ce qui concerne la traduction, on sait que chez le sujet parkinsonien, la dégénérescence du système dopaminergique tubéroinfundibulaire, parallèlement à celle du système nigrostriatal, n'affecte pas les taux de peptides dérivés de la POMC dans l'hypothalamus [23]. Bien que chez le rat, plusieurs travaux suggèrent une diminution, médiée par la dopamine, des contenus hypothalamiques en $\beta$-endorphine, il est démontré que les neurones dopaminergiques n'affectent pas la biosynthèse de la POMC et de ses dérivés dans le SNC des mammiferres.

c) Régulation post-traductionnelle. De récents travaux suggèrent que la dopamine pourrait contrôler la maturation posttraductionnelle des peptides dérivés de la POMC. Ainsi, la dopamine exerce un tonus inhibiteur sur la biosynthèse de l'opiomélanotropine acétyltransférase (OMAT), sans modifier les caractéristiques biochimiques et cinétiques de cette enzyme. Le fait que la dopamine bloque, chez les amphibiens, la libération de l' $\alpha$-MSH monoacétylée [16] est en accord avec la réduction de l'activité OMAT mentionnée précédemment. A l'inverse, chez le rat, le groupe de Smyth [17] suggère que le traitement à la dopamine provoque la formation de la $\beta$-endorphine $\mathrm{N}$ acétylée dans l'hypophyse et le mésencéphale, mais non dans l'hypothalamus [17]. La dopamine a donc pour effet de diminuer dans tous les cas l'activité intrinsèque des peptides dérivés de la POMC.

d) Contrôle de la libération des neuropeptides dérivés de la POMC. L'étude du processus de libération des dérivés de la POMC a été entreprise, in vitro, dans un système de périfusion* de tranches hypothalamiques de rat. La perfusion d'agents dépolarisants induit une stimulation rapide et significative de la sécré- tion d' $\alpha-\mathrm{MSH}$ et de $\beta$ endorphine. Ces effets sont totalement abolis en l'absence de calcium. Le contrôle éventuel de la libération de ces neuropeptides par la dopamine, a été étudié par la même approche. La dopamine inhibe la libération de $\beta$ endorphine stimulée par le potassium, mais elle n'a pas d'effet sur celle de l' $\alpha$-MSH. On se rend compte que les arguments en faveur d'une action inhibitrice dopaminergique sur l'activité des neurones à POMC de l'hypothalamus médiobasal sont largement controversés que ce soit au niveau de la biosynthèse du précurseur ou de la libération de ses dérivés. Par ailleurs, une déplétion cérébrale sérotoninergique provoque une baisse des taux tissulaires de $\beta$-endorphine, d' $\alpha$-MSH et d'ACTH dans l'hypothalamus et le tronc cérébral. Bien que le niveau de régulation ne soit pas encore précisé, l'hypothèse d'un contrôle sérotoninergique est confirmée par l'existence de contacts synaptiques entre les fibres à sérotonine et à ACTH [20].

e) Dégradation synaptique des dérivés de la POMC. Les peptides du groupe POMC libérés dans la fente synaptique peuvent être inactivés comme le sont les neurotransmetteurs traditionnels ; à la suite de modifications chimiques spécifiques, ils peuvent également générer des peptides avec

- Lo technique de périfusion consiste à incuber des fragments de tissu (dans l'étude citée ici, ce sont des tranches d'hypothalamus de rat) dans un flux continu et unidirectionnel de milieu de culture. Le modèle de périfusion présente plusieurs avantages sur les techniques d'incubation statique conventionnelles. Cetle méthode évite en particulier l'accumulation des neuropeptides dans le milieu d'incubation, ce qui pourrait entraîne une autorégulation artéfactuelle. D'autre part, la mesure en continu des produits libtrés permet de suivre la cinétique de la réponse neuronale à divers stimuli. En revanche, la technique de périfusion nécessite des méthodes de détection très sensibles puisque les neuropeptides se trouvent instantanément dilués par l'approvisionnement continu en milieu de culture. Ainsi, les taux d' $\alpha-M S H$ libérée par un hypothalamus de rat est d'environ 0,1 fMole $\left(10^{-16}\right.$ Mole) par minute. Seuls des radioimmunoétalonnages très sensibles permettent de mesure les quantités infimes de neuropeptides libérés dans ces conditions. des activités différentes. L' $\alpha$-MSH est clivée dans l'espace synaptique par une variété d'amino- et d'endo-peptidases en fragments biologiquement inactifs. Le groupement $\alpha$-N-acétyl confere à l' $\alpha$ MSH une résistance particulière à ces enzymes de dégradation et potentialise son effet physiologique. L'inactivation de la $\beta$ endorphine (1-31) peut résulter soit de sa maturation intraneuronale qui conduit aux $\beta$-endorphines $1-27$ et $1-26$, soit de sa dégradation dans la fente synaptique. Or, la dégradation intraneuronale de la $\beta$-endorphine, plus qu'un simple processus d'inactivation des effets analgésiques, conduit à la formation de $\beta$-endorphine (1-27) qui présente une activité antagoniste des opiacés quatre fois plus puissante que celle de la naloxone. Toutefois, la dégradation de la $\beta$ endorphine (1-31) en (1-27) est mineure, car la conformation de la $\beta$-endorphine la protège à ses extrémités $\mathrm{N}$ - et $\mathrm{C}$-terminales. $\mathrm{La}$ $\beta$-endorphine oriente son clivage de façon prépondérante vers la formation des $\gamma^{-}$et $\alpha$ endorphines. La protéolyse de la $\beta$-endorphine dans la fente synaptique entraîne donc : 1) l'abolition de l'activité opiacée de la $\beta$ endorphine et 2) la production de la $\gamma$-endorphine et de la destyr'- $\gamma$-endorphine qui possèdent des activités neuroleptiques [24], ou de l' $\alpha$-endorphine et ses dérivés qui ont des activités psychostimulantes [25].

$f$ Sites de liaison des peptides dérivés de la POMC. Grâce aux études pharmacologiques et biochimiques, les récepteurs aux opiacés sont classés en trois types suivant leur affinité pour les différents peptides opiacés : les sites mu, delta et kappa. Ainsi, la morphine se lie aux sites mu, la $\beta$-endorphine aux sites mu et delta alors que les enképhalines se fixent sur les sites delta. A l'heure actuelle, les sites de liaison de l' $\alpha$ MSH dans le cerveau n'ont pas pu être étudiés, du fait de leur très faible concentration et de la difficulté à obtenir un ligand marqué spécifique. Il est raisonnable 
de postuler l'existence de récepteurs à l' $\alpha$-MSH compte tenu des nombreuses actions psychotropes de ce neuropeptide. La découverte récente de nouveaux agonistes de l' $\alpha$-MSH tels que le [Nleu ${ }^{4}, \mathrm{D}-$ $\left.\mathrm{Phe}^{7}\right]-\alpha-\mathrm{MSH}$ et l'Ac-[Cys${ }^{4-}$ $\left.\mathrm{Cys}^{10}\right]-\alpha-\mathrm{MSH}$ résistants à la dégradation et plus actifs que l' $\alpha$ MSH naturelle, devrait prochai- nement conduire à la caractérisation des récepteurs de l' $\alpha$-MSH.

\section{Effets}

physiologiques centraux

$\alpha$-MSH et ACTH. L' $\alpha$-MSH est impliquée dans le contrôle de l'éveil, la motivation, l'attention, l'apprentissage et l'agressivité [1].
L'ACTH, l' $\alpha$-MSH et les peptides apparentés retardent l'extinction du comportement d'évitement** et facilitent celui d'approche motivé par la faim. Face à de nouvelles situations, les rats traités par l' $\alpha$-MSH se repèrent plus vite dans un labyrinthe. Par ailleurs, ce neuropeptide facilite l'apprentissage des discrimina-

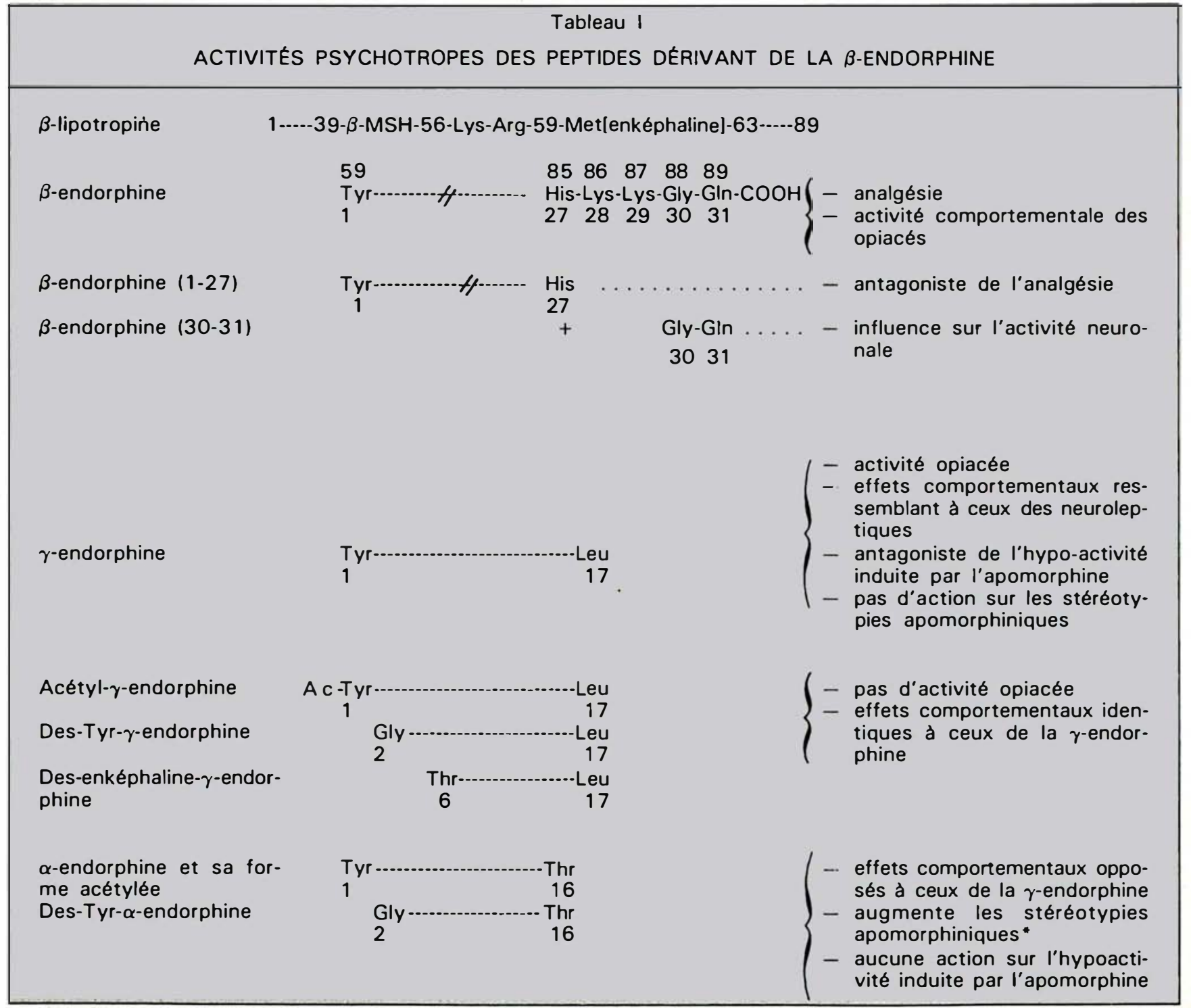

- Les stéréotypies apomorphiniques sont des comportements spécifiques et inéluctables induits par les agonistes dopaminergiques. Elles ont une origine centrale et se caractérisent, selon les espèces, par des crises de vomissement, des contractions de la máchoire, des reniflements et un toilettage excessif. 
tions visuelles et renforce chez le rat le processus de motivation et d'attention qui est accompagné d'une diminution significative de l'anxiété.

Endorphines. L'administration centrale de $\beta$-endorphine entraîne les effets caractéristiques des opiacés : une analgésie prolongée, une tolérance, une perte de la catalepsie engendrée par les neuroleptiques et une immobilité chez le rat, qui a pu être comparée à une catatonie. Ces phénomènes sont tous réversés par la naloxone. La $\beta$-endorphine agit également sur les fonctions neuroendocriniennes via les neurones à POMC du noyau arqué et/ou amygdalien, chez l'homme et le rat [26]. Elle inhibe la sécrétion de l'hormone lutéotrope et stimule, indirectement, celle de prolactine et d'hormone de croissance, en déprimant le tonus inhibiteur du système dopaminergique tubéroinfundibulaire. Les $\gamma$ - et $\alpha$-endorphines, qui sont des produits de dégradation synaptique de la $\beta$-endorphine, semblent avoir une action centrale indépendante des récepteurs aux opiacés (Tableau I). La $\gamma^{-}$et la des-tyr ${ }^{1}-\gamma$-endorphine ont des effets similaires aux neuroleptiques, alors que les propriétés de $l^{\prime} \alpha$-endorphine se rapprochent de celles de l'amphétamine [25]. Compte tenu de l'activité neuroleptique de la des-tyr'- $\gamma$-endorphine, ce peptide a été administré chez certains schizophrènes par l'équipe de De Wied [27] : un pouvoir antipsychotique a été noté chez les patients atteints de troubles légers alors que les performances des schizophrènes chroniques n'ont pas été améliorées. Le clivage de la $\beta$-endorphine peut donc donner naissance à une variété de peptides actifs sur le système nerveux central. Il est intéressant de souligner que les effets psychotropes des dérivés de la $\beta$-endorphine sont différents entre eux, voire même opposés (Tableau I).

Interaction $\alpha$-MSH et $\beta$ endorphine. L' $\alpha$-MSH déacétylée antagonise l'analgésie induite par la morphine et la $\beta$-endorphine, alors que la $\beta$-endorphine peut $m / s$ n० 3 vol. 3 , mars 87 moduler l'effet de l' $\alpha$-MSH sur les mélanocytes. Cette dernière activité est portée par la portion $\mathrm{C}$-terminale de la $\beta$-endorphine, région qui ne contient pas d'activité opioïde. Dans le système nerveux central, l'interaction des $\alpha$ MSH et $\beta$-endorphine avec leurs récepteurs est encore modulée par la $\gamma$-MSH, antagoniste de ces deux dernières [28].

\section{Conclusion}

Ces quelques données montrent la complexité des systèmes neuronaux à POMC. Ceux-ci synthétisent et libèrent une variété de peptides possédant des effets psychotropes multiples et parfois antagonistes. L'activité de ces neurones semble être la résultante d'une multitude de facteurs présynaptiques et synaptiques agissant sur la nature et le taux des neuropeptides libérés. Les systèmes neuronaux à POMC sont donc des modèles exceptionnels pour l'étude des fonctions multineurotransmettrices des neurones peptidergiques dans le SNC

- Le comportement d'évitement consiste en la fuite provoquée par un stimulus nociceptif appelé aussi stimulus inconditionnel (inné). Ce stimulus est en général provoqué par une décharge électrique dans les pattes de l'animal au moyen d'un plancher grillagé électrifié. Si le stimulus inconditionnel est précédé d'un stimulus sensoriel conditionnel (lumière colorée, sonnerie) l'animal associe les deux stimuli et réagit par la fuite. Apres la phase d'acquisition, la stimulation conditionnelle suffit à elle seule à provoquer la réaction de fuite. Les psychotropes modifient les réponses d'évitement de l'animal. Ainsi, les neuroleptiques entraînent le déconditionnement, alors que les hypnotiques font disparaitre le réflexe d'évitement aux stimuli conditionnel et inconditionnel.

\section{Remerciements}

Ce travail a bénéficié de l'aide du CEA-ORIS (contrat 098-539), de la DRET (contrats 85-1406, 86-1375 et 86-1164), de l'Inserm (contrats 82-4019, 84-6020 et 86-4016), du MEN (86-1128), de l'Institut de Recherches Internationales SER VIER et du Ministère des Relations Extérieures (Projet sur Thème France-Québec).

\section{Summary}

Proopiomelanocortin (POMC) is a glycoprotein which serves as a multihormonal precursor for ACTH, LPHs, MSHs and endorphins. This precursor protein is synthetized in corticotrophs and melanotrophs of the pituitary, but it is also present in the central nervous system, where three groups of perikarya containing various POMC-related peptides have been identified : the arcuate nucleus (AN) and dorso-lateral area of the hypothalamus, and the nucleus of the tractus solitarius of the mesencephalon. Processing of POMC in the cell bodies of the AN follows a similar pattern as in intermediate lobe cells. Endopeptidases cleave the prohormone at paired basic amino acid residues and generate two major mature products : $\alpha$-MSH and $\beta$-endorphin. Then, enzymatic modifications, including $\mathrm{N}-\alpha$ acetylation and C-terminal amidation, determine the biological activity of these peptides. The mechanisms regulating the activity of POMCcontaining neurons are still unknown. Transcription is not altered by glucocorticoids, adrenalectomy or dopamine ; serotonin seems to stimulate translation or processing while a possible control of POMC neurons by dopamine is extremely controversial. The intraneuronal localization of POMC-derived molecules and the numerous behavioral effects of these peptides suggest that they play neurotransmitter functions. POMC neuronal systems make exceptional models to study the functioning of peptidergic neurons.

\section{TIRÉS A PART}

H. Vaudry : UA Cnrs 650, Unité alliée à l'Inserm, faculté des sciences, université de Rouen, 76130 Mont-Saint-Aignan. 